\title{
Fast Identification of Control Signaling Aided by Please-Decode-Blindly (PDB) Messages
}

\author{
Reza Moosavi and Erik G. Larsson
}

\section{Linköping University Post Print}

N.B.: When citing this work, cite the original article.

C2012 IEEE. Personal use of this material is permitted. However, permission to reprint/republish this material for advertising or promotional purposes or for creating new collective works for resale or redistribution to servers or lists, or to reuse any copyrighted component of this work in other works must be obtained from the IEEE.

Reza Moosavi and Erik G. Larsson, Fast Identification of Control Signaling Aided by PleaseDecode-Blindly (PDB) Messages, 2012, IEEE Swedish Communication Technologies Workshop (Swe-CTW), 24-26 October, Lund, Sweden.

http://dx.doi.org/10.1109/Swe-CTW.2012.6376289

Postprint available at: Linköping University Electronic Press

http://urn.kb.se/resolve?urn=urn:nbn:se:liu:diva-82104 


\title{
Fast Identification of Control Signaling Aided by Please-Decode-Blindly (PDB) Messages
}

\author{
Reza Moosavi and Erik G. Larsson
}

\begin{abstract}
Blind decoding of control information is used in some wireless multiple access systems such as LTE to achieve adaptive modulation and coding, as well as to address the multiple access problem on the control channel. Blind decoding incurs high computational complexity in mobile terminals. In this paper, we describe a scheme to reduce the computational complexity associated with the blind decoding. The main idea is to broadcast a "please-decode-blindly" message to all terminals that are eligible for scheduling, to instruct a subset of the terminals to perform the blind search. We propose two schemes to implement our idea and we investigate their performances via system simulations.
\end{abstract}

Index Terms-Blind decoding, Adaptive coding and modulation, Control signaling

\section{INTRODUCTION}

In many systems such as 3rd Generation Partnership Project (3GPP) Long-Term Evolution (LTE), the control information including the information regarding how the resources are allocated among the terminals, is sent to the terminals at the beginning of each so-called subframe. The reason for doing that is twofold: (i) the scheduled terminals would know where they are allocated as early as possible, and hence they would be able to process the data region before the end of the subframe, and (ii) the terminals that are not scheduled in the subframe are able to power down their front-end receiving circuits during the transmission of the subframe and hence they can save energy [1].

The control information of different terminals is first encoded using a terminal specific cyclic redundancy check (CRC) and then mapped onto the control channel. This enables the terminals to find their control information blindly. More precisely, in order to find the control information, a terminal needs to decode the control information blindly for different combinations of channel codes and/or different combinations of the location in the control region, and for each such combination, to check whether the CRC checks or not. In the case that the CRC checks, then the terminal assumes that the control information was intended for it and that it was decoded correctly. In the case that the CRC does not check for any of the possible combinations, then the terminal assumes that it has not been scheduled for reception of payload data

The authors are with the Dept. of Electrical Engineering (ISY), Linköping University, Linköping, Sweden. Email: $\{$ reza,egl $\} @$ isy.liu.se. This work was supported in part by Ericsson, VINNOVA and the Excellence Center at Linköping-Lund in Information Technology (ELLIIT). in the current subframe. It is worth noting that the blind decoding process described above in addition to achieving adaptive modulation and coding (AMC), solves the multiple access problem on the control channel via the terminal specific CRC.

Blind decoding incurs a high computational complexity on the terminals. This is because a terminal might need to try many blind decoding attempts before it finds the control information. In order to reduce the computational complexity of blind decoding in LTE, the number of blind attempts performed by each terminal is limited. More precisely, a terminal only monitors some specific parts of the control region, which is known as the search space, for possible control information. It is worth noting that this puts an extra constraint on the scheduling problem as well. This is because if a certain terminal is going to be scheduled for the reception of payload data, the base station needs to make sure that the corresponding control information for that terminal (which contains the scheduling assignment), can be accommodated in its search space, since otherwise the terminal will not find its payload data [1, Section 10.4.9]. Since the terminals typically have limited battery, any reduction in the computational complexity of blind decoding is valuable. Additionally, by reducing the computational complexity of blind decoding, we can expand the search spaces of the terminals and hence resolve the above accommodation problem too.

In this paper, we propose a scheme to decrease this computational complexity. Note that in the existing blind decoding scheme all the terminals, regardless of whether they have been scheduled or not, need to try to decode the incoming control information blindly to find out whether they are scheduled and if so, determine where their payload data is located in the subframe. We are interested in finding ways of signaling the control information such that only the terminals that have been scheduled in the subframe and hence benefit from doing the blind search attempt, perform the blind decoding process and those that have not been granted any resource in the subframe can power down their front-end circuits immediately without performing any blind decoding attempts.

The main idea of our scheme is to broadcast a "pleasedecode-blindly" (PDB) message to all the terminals that are eligible for scheduling, to instruct specific groups of terminals to perform the blind search. Ideally, the addressed group should only consist of the terminals that are scheduled in the subframe. We will describe two different schemes for reducing the number of blind search attempts in Sections IV and V and 
we will exemplify their performances using system simulations in Section VII.

\section{RELATED WORK}

This work is motivated by the radio resources cost that is needed for the transmission of control information [2][4]. As it has been shown therein, this signaling cost can be substantial in many situations. Blind decoding, described above, is a way to reduce the signaling cost since it allows the use of adaptive modulation and coding for the transmission of the control information. We are aware of relatively little literature addressing the computational complexity problem of blind decoding. For example, in [5] a method for reducing the number of blind attempts performed by the terminals was proposed. The idea is to limit the number of combinations in which the control information may be arranged and located in the control region, which in turn results in a search space reduction. In [6], another scheme to decrease the number of blind decoding attempts was proposed. The idea therein is to decode the received data partially according to each combination in the search space and terminate the ones that yield low quality metrics. The quality metric can be cumulative log-likelihood ratios for instance. In [7], a scheme for fast blind detection of channel codes was proposed. This is done by evaluating the syndrome posterior probabilities obtained from the parity check matrix of each candidate channel code and to pick the code that achieves the highest syndrome posterior probability. In [8] a solution to decrease the detection errors of blind decoding in LTE was proposed. The proposed scheme therein works by modifying the circular buffer used to read out the coded bits, in such a manner that the control information can be decoded unambiguously by the receiving terminals. In [9], a new scheme for reduction of blind decoding complexity in LTE was suggested. The idea is to use a mini-CRC injected early in the data stream to determine if the current decoding attempt is using the correct modulation and coding parameters, which ultimately reduces the complexity of the blind decoding process.

\section{PROPOSED SCHEME}

The main idea of our proposed scheme is to broadcast a "please-decode-blindly" (PDB) message to all the terminals that are eligible for scheduling, to instruct specific groups of terminals to perform the blind search. Ideally, the addressed group should only consist of the terminals that are scheduled in the subframe and hence benefit from doing the blind decoding search attempt. In practice to limit the number of groups, each group may consist of the other terminals as well. The interpretation of doing this is that only a subset of the terminals for whom there is payload data transmission during the subframe, will perform the blind decoding search and hence the number of total blind searches will be decreased compared to the conventional way of control signaling. Note that all the terminals should be able to receive and decode the PDB message without doing blind search. Therefore, the PDB message should be broadcast at the very beginning of each subframe and the terminals should know where to look for it. ${ }^{1}$ Thus, all the terminals listen to the PDB message and only those that are addressed via the PDB message will perform the blind decoding attempt. The key element is therefore how the groups are formed. We propose a grouping scheme based on the traffic status of the terminals, and where the groups are nested. The point of nesting the groups is that a large set of them can easily be addressed simultaneously. Moreover, it should be possible to move the terminals between different groups when the network traffic pattern changes, and this should be done without incurring additional control signaling. That is, the grouping assignments should be a function which can be computed by both the individual terminals and by the base station.

Let $K$ denote the total number of groups and let there be $N_{u}$ terminals. In mathematical terms, we are interested in finding a mapping

$$
f: \mathcal{S} \longmapsto\{1,2, \ldots, K\}
$$

such that the overall number of blind searches is minimized. Herein $\mathcal{S}$ is the set consisting of all $2^{N_{u}}$ possible subsets of the terminals. In the ideal case, having $K=2$ is sufficient for achieving the minimum number of blind search attempts in each scheduling interval with the mapping $f$ defined as

$$
f:\left\{\boldsymbol{S}, \boldsymbol{S}^{c}\right\} \longmapsto\{1,2\},
$$

with $f(\boldsymbol{S})=1$, and $f\left(\boldsymbol{S}^{c}\right)=2$, where $\boldsymbol{S}$ denotes the set of terminals that are scheduled for payload reception in the subframe, and $S^{c}$ is the complement of this set, i.e. the set of terminals that are not scheduled for payload reception in the subframe. In this case, by sending the PDB message " 1 " only the scheduled terminals will perform the blind search. The total number of blind searches will be $|\boldsymbol{S}|$, which is the minimum possible value.

In practice the problem of finding the optimum grouping as well as how to assign/move terminals between groups is hard. This is because (i) the set of scheduled terminals $\boldsymbol{S}$ is not fixed over time and is changing from subframe to subframe, and (ii) the mapping $f$ should be known to the terminals without incurring additional signaling overhead. We note that there are many ways that the grouping can be done. From a source coding standpoint, it is desirable that all the groups are equally likely to be chosen. However, the main focus in this study is to decrease the terminals' energy consumption by reducing the number of blind decoding attempts they have to perform for finding their respective control information. Therefore, we are interested in finding a grouping in such a way that the terminals that are more likely to be scheduled in the subframe perform the blind decoding search more often than those that are less likely to be scheduled. In the next two sections we will present two different ways of doing the grouping.

\footnotetext{
${ }^{1}$ The PDB message is thus reminiscent of Physical Control Format Indicator Channel which is used to signal the length of the control region in LTE.
} 


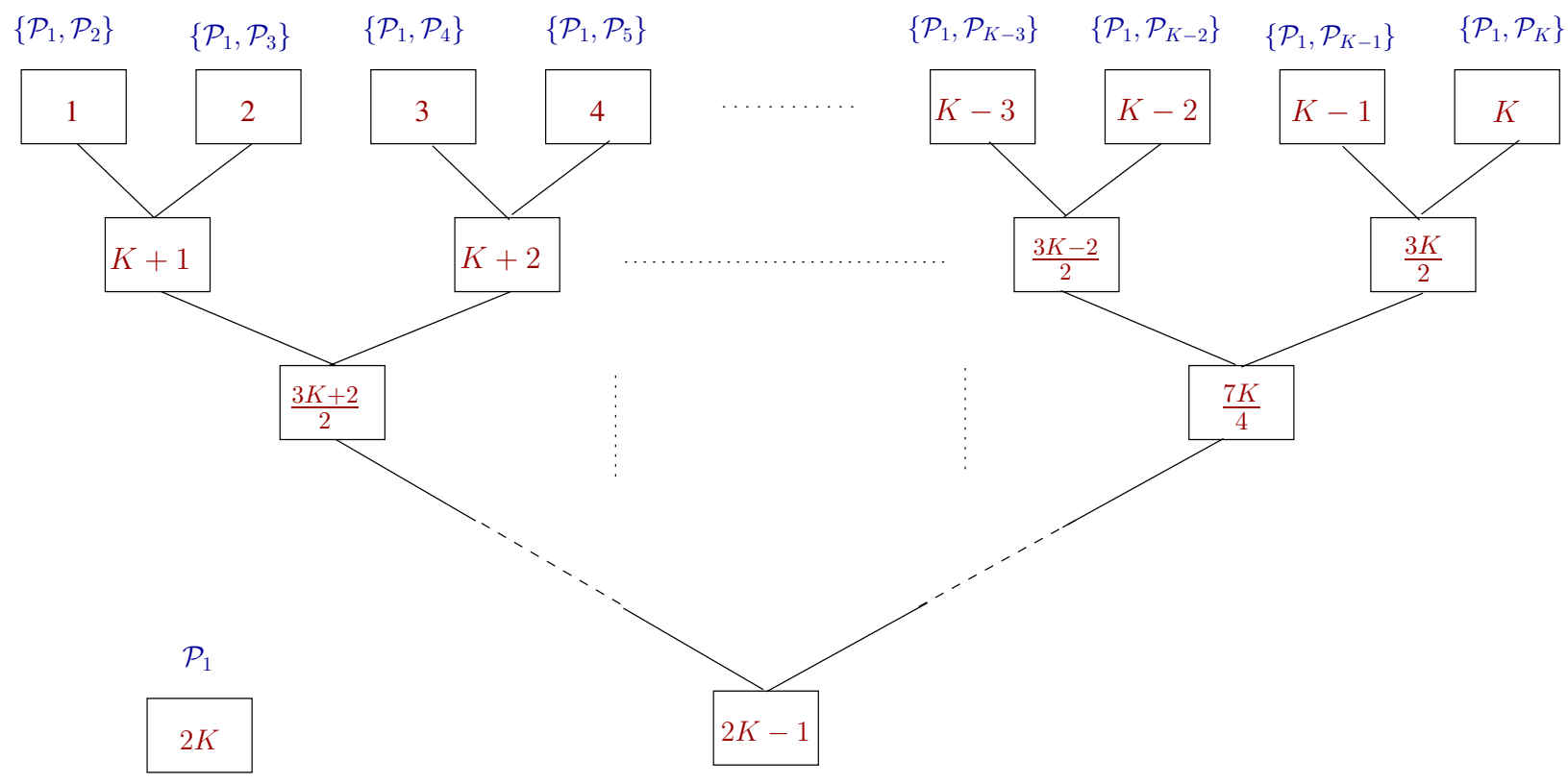

Fig. 1. Groups and their relations with the value announced by the PDB control message. Herein, the assumption is that $K=2^{C}$ for some integer $C$.

\section{Grouping BASED on the Assignments History}

In this scheme, we define the groups based on the terminals' assignments history in the past $M$ subframes $(M \geq K)$. More precisely, we define group $\mathcal{P}_{1}$ to consist of the terminals that have been scheduled in at least $K-1$ subframes, group $\mathcal{P}_{2}$ to consist of the terminals that have been scheduled in exactly $K-2$ out of the previous $M$ subframes and so forth. Group $\mathcal{P}_{K}$, also denoted by the "idle group", comprises the terminals that have not been scheduled in the previous $M$ subframes. For simplicity of the notation, assume that $K=2^{C}$ for some integer $C$. Based on these groups, we form a tree as in Fig. 1. At the beginning of the subframe, a PDB control message of length $\log _{2}(2 K)=1+C$ is broadcast indicating one of the nodes in the tree. Once the control message is received, all the terminals in leaf groups that are connected to the given PDB message are instructed to perform the blind decoding attempt. For instance, if control message $K-2$ is sent, ${ }^{2}$ then the terminals in groups $\mathcal{P}_{1}$ and $\mathcal{P}_{K-2}$ perform blind decoding search and if control message $K+2$ is received, then the terminals in groups $\mathcal{P}_{1}, \mathcal{P}_{4}$ and $\mathcal{P}_{5}$ perform blind decoding attempt. Finally, control message $2 K-1$ which corresponds to the root node, indicates that all the terminals shall perform blind search. Also we see that the "active terminals" i.e. the terminals in the group $\mathcal{P}_{1}$, always perform blind search. If control message $2 K$ is sent, then only the active terminals perform blind decoding attempt.

\section{Grouping Based on the Traffic Status}

For this scheme, we define different traffic zones and we assign the terminals to these traffic zones. We further assume the following:

\footnotetext{
${ }^{2}$ note that this PDB corresponds to a leaf node.
}

- At the beginning of each subframe, a PDB control message of length $\left\lceil\log _{2} K\right\rceil$ is broadcast to all the terminals, indicating one of the $K$ traffic zones.

- All terminals that are assigned to the traffic zones less than or equal to the "announced" traffic zone by the PDB message will do the blind search for finding the possible control data.

- If a terminal is scheduled for reception of payload data, then it moves to the zone below its current one, unless it is already in the lowest traffic zone. That is, if the terminal is in the traffic zone $k$ and if it is assigned some resources in the subframe, then it will move to the traffic zone $k-1$ for the upcoming subframes, unless $k=1$ already.

- If a terminal is not scheduled for reception of any payload data for a specific period, say for $L$ consecutive subframes, then it moves to a higher traffic zone, unless it is already in the highest traffic zone. In other words, if the terminal is in the traffic zone $k$ and is not scheduled for $L$ consecutive subframes, then it will move to the traffic zone $k+1$, unless $k=K$ already.

With this scheme, the terminals are assigned to the traffic zones based on their respective buffer states. More precisely, terminals with large buffer sizes are assigned to the lower zones and vice versa. In the beginning of each subframe, a traffic zone is announced and the terminals that are in zones higher than the announced zone will power down their frontend decoding circuits immediately without performing any blind attempt.

Ideally, the scheduler should keep the "active" terminals (terminals awaiting payload data) in traffic zone 1 and "idle" terminals (terminals with no payload data) in traffic zone $K$. If the scheduler needs to send payload data to a specific terminal, 
say terminal $\ell$, which is in the traffic zone $k$, then traffic zone $k$ is announced at the beginning of the subframe, making all terminals in traffic zones less or equal than $k$ perform a blind decoding search. Then terminal $\ell$ will move to traffic zone $k-1$ for the next subframe, and will eventually move to the first traffic zone. On the other hand, if one of the active terminals, say terminal $\ell^{\prime}$, has no more payload data, then it will consequently move to higher zones, due to the fact that it will not be scheduled for the reception of payload data. This process describes how the terminals are assigned to the traffic zones and how the zone assignments are changed over time based on their respective traffic patterns.

We see that the first scheme described in Section IV does not rely on the traffic pattern, and the nesting is done through forming the groups based on a tree structure. This scheme is therefore suitable for the situations where no a priori information is available on the incoming traffic. On the other hand, the second scheme depends more on specific assumptions on the traffic model, and is useful for scenarios where the payload information for the terminals come in a burst fashion.

\section{THEORETICAL JUSTIFICATION}

To see how the above proposed schemes work and how far their corresponding total average number of blind attempts are compared to the optimum situation, let us consider a simple system. Suppose that the payload data for individual terminals is arriving independently of one another, with a given probability $p$. Once some payload data for a terminal arrives, the terminal will be scheduled immediately for the coming $N$ subframes, until all its data has been sent completely. In other words, there are enough resources to schedule all the active terminals and each active terminal requires exactly $N$ subframes to receive all its data. This model represents a system where the payload traffic for individual terminals has a fixed packet size, and where the arrival of new packets is a random variable with a Poisson distribution with a fixed rate, which is a relevant model for many practical systems. However, this model does not consider the scarcity of resources in the scheduling since we assume that there are always enough resources to accommodate all active terminals. This assumption is not true especially if the number of terminals is large. However, we may justify this by considering a larger $N$ in those situations.

For the first scheme (Section IV), assume that $M=K=2$. That is, the grouping is performed based on the terminals previous subframe assignments as follows: If a terminal has been scheduled in the previous subframe, then it will be assigned in group $\mathcal{P}_{1}$, otherwise it will be assigned in group $\mathcal{P}_{2}$. For the second scheme (Section V), let us assume that there exists two traffic zones, with $L=1$.

With these parameters, the two schemes coincide. Let us first find the probability that a terminal is in the active state. Based on the given model, we can sketch the state diagram as given by the Markov chain in Fig. 2. According to Fig. 2, a terminal is active if it is not in state $I$. The probability of

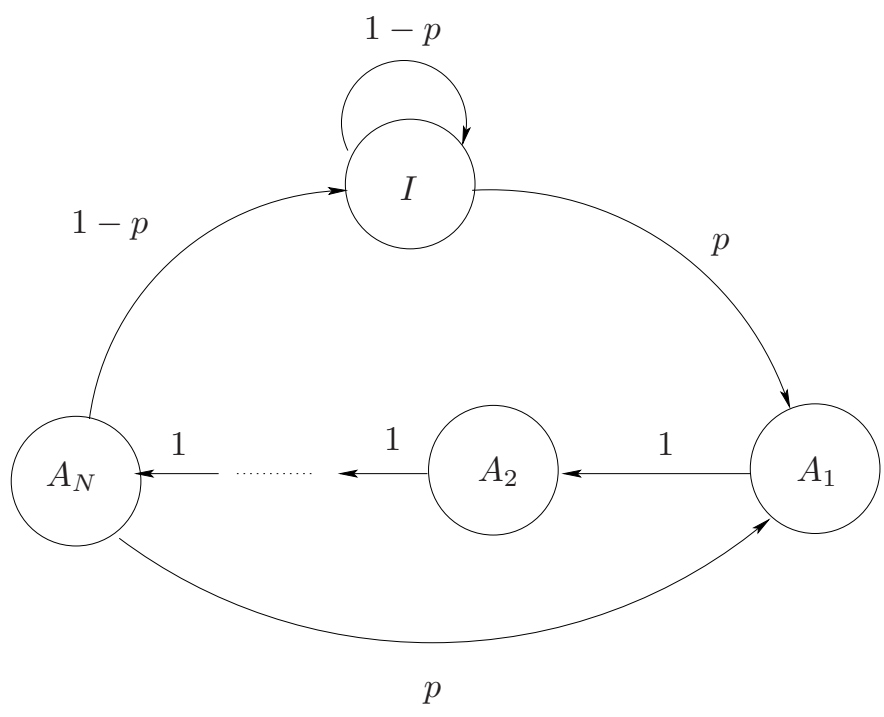

Fig. 2. State diagram for mobile terminals.

being in state $I$ is

$$
p_{I} \triangleq \operatorname{Pr}\{\text { Idle State }\}=\frac{1-p}{1+p(N-1)} .
$$

Ideally, the total number of blind search attempts is equal to the number of active terminals, i.e. number of terminals awaiting payload data reception. Thus for a system with $N_{u}$ terminals, the average number of blind search attempts is ideally $N_{u}\left(1-p_{I}\right)$. Let $\mathcal{A}(i)$ denote the event that there are exactly $i$ active terminals. We have

$$
\operatorname{Pr}\{\mathcal{A}(i)\}=\left(\begin{array}{c}
N_{u} \\
i
\end{array}\right)\left(1-p_{I}\right)^{i} p_{I}^{N_{u}-i} .
$$

For our proposed schemes, the arrival of payload data for any of the idle terminals triggers a blind search attempt for all $N_{u}$ terminals. Let $\mathcal{B}(i)$ denote the event that payload data arrives for one or more terminals from a total of $i$ idle terminals. We have,

$$
\begin{aligned}
\operatorname{Pr}\{\mathcal{B}(i)\} & =1-\operatorname{Pr}\{\text { No data for any of the } i \text { idle terminals }\} \\
& =1-(1-p)^{i},
\end{aligned}
$$

and thus the average number of blind decoding attempts, if there are $i$ idle terminals, is

$$
\begin{aligned}
T(i) & =N_{u} \operatorname{Pr}\{\mathcal{B}(i)\}+\left(N_{u}-i\right)(1-\operatorname{Pr}\{\mathcal{B}(i)\}) \\
& =N_{u}-i(1-p)^{i} .
\end{aligned}
$$

Therefore, the average number of blind attempts for our proposed schemes is,

$$
\begin{aligned}
\sum_{i=0}^{N_{u}} T(i) \cdot \operatorname{Pr}\left\{\mathcal{A}^{c}(i)\right\} & =\sum_{i=0}^{N_{u}} T(i) \cdot\left(\begin{array}{c}
N_{u} \\
i
\end{array}\right) p_{I}^{i}\left(1-p_{I}\right)^{N_{u}-i} \\
& =N_{u}\left(1-p_{I}(1-p)\left(1-p p_{I}\right)^{N_{u}-1}\right) .
\end{aligned}
$$

As it can be seen, the total number of blind decoding 
attempts saved according to our schemes compared to the conventional approach, where all terminals perform blind search regardless of being scheduled or not, is $N_{u} p_{I}(1-$ $p)\left(1-p p_{I}\right)^{N_{u}-1}$ on the average. For the ideal case, the savings is however $N_{u} p_{I}$. Since $\left(1-p p_{I}\right)^{N_{u}-1}$ approaches zero as the number of terminals grows, one may expect that the savings according to our schemes diminishes for large values of $N_{u}$. However, for practical systems $p$ is usually small and additionally, the number of users is not large. Therefore, by using the approximation

$$
(1-x)^{N} \approx 1-N x
$$

for small $x$ and moderate values of $N$, we expect savings of roughly $N_{u} p_{I}(1-p)\left(1-\left(N_{u}-1\right) p p_{I}\right)$ with our proposed schemes.

\section{NUMERICAL ILLUSTRATION}

In this section, we provide some numerical examples of how our proposed schemes work for practical systems. As a performance measure, we count the average number of blind searches according to each scheme and compare it with the optimal case, where only the scheduled terminals perform the blind search.

\section{System Model}

We consider an LTE-like OFDMA system with $5 \mathrm{MHz}$ bandwidth corresponding to 300 OFDM subcarriers (plus some guard subcarriers). We assume that the scheduling decision is made at the beginning of each subframe of duration 1 msec, consisting of 14 OFDM symbols. The scheduling granularity, denoted by a resource block, is assumed to be 14 OFDM symbols in time and 12 consecutive OFDM subcarriers in frequency, as in LTE [1]. With the given parameters, there are 25 resource blocks in each subframe. We further assume that the terminals have independent and identically distributed (i.i.d.) Rayleigh fading channels with mean square value equal to 1 . If a certain resource block, say resource block $n$, is assigned to terminal $i$, we assume that

$$
C_{n}(i)=\sum_{(t, f) \in \mathcal{T}^{n}} \log _{2}\left(1+\delta\left|\alpha_{t, f}^{i}\right|^{2} \Gamma\right)
$$

bits will be sent to the terminal, where $\left|\alpha_{t, f}^{i}\right|$ represents the channel gain of terminal $i$ in OFDM symbol $t$ and subcarrier $f, \Gamma$ is the average signal-to-noise-ratio (SNR), and $\delta$ denotes the capacity gap to the Shannon capacity formula [10]. Also $\mathcal{T}^{n}$ denotes the set of all OFDM subcarriers and symbols in resource block $n$.

\section{Traffic Model}

We consider a best effort traffic model representing a webbrowsing type of application [11]. We use the arrival model in [12] for the incoming traffic. More precisely, the data packet size is drawn according to truncated Pareto distribution with a minimum and a maximum sizes of 8.5 Kbytes and 2 Mbytes respectively. The application inter-arrival time is geometrically distributed with a mean of 5 seconds and takes on values that are multiples of $1 \mathrm{sec}$. With these parameters, the mean size of the application packets is 34 Kbytes and the average traffic arrival rate is $54.4 \mathrm{kbps}$. Let $N(i)$ denote the total number of bits that need to be transmitted to terminal $i$, drawn according to the given model.

\section{Scheduling Rule}

Let $S_{n}$ denote the terminal that has been scheduled in resource block $n$. We assume the following scheduling rule:

$$
S_{n}=\underset{i}{\operatorname{argmax}} N(i) \cdot C_{n}(i) .
$$

The reason for choosing this scheduler is to guarantee some amount of fairness among the terminals while at the same time exploit multi-user diversity to some extent. As mentioned earlier, if resource block $n$ is assigned to terminal $i$, we assume that $C_{n}(i)$ bits will be sent to terminal $i$ during the payload transmission.

\section{Simulation Results}

Fig. 3 illustrates the average number of blind attempts for different schemes. We choose the parameters such that both schemes require the same amount of signaling overhead. More precisely, a PDB message of length 2-bits is required for the two schemes, corresponding to 2 and 4 groups according to scheme I and scheme II respectively. We further assume that $M=9$ and $L=1$. The average SNR is set to be $5 \mathrm{~dB}$ in this case with $\delta=1 / 2$. As we can see, a saving of $50 \%$ is possible according to both schemes compared to the conventional approach. To compare the results with the theoretical predictions given in Section VI, we also plotted the expected number of blind attempts given by (6), with the nominal values for $p$ and $N$ obtained from the mean values that have been used for the traffic model as follows. Since the inter-arrival time between packets has a geometric distribution with mean 5 seconds, and since the duration of a subframe is 1 msec, we assume $p=2 \times 10^{-4}$. To find $N$, we use the mean value for the packet size (34 Kbytes) and we assume that the resources in each subframe is distributed evenly among the terminals and each resource can carry $C_{n}(i)$ bits with $\left|\alpha_{t, f}^{i}\right|^{2}=1$. Note that since $p$ is small, the average number of blind attempts according to (6) is roughly $N_{u} p_{I}$ which is the same as the optimum value obtained by assuming the simple model in Section VI.

As can be seen from Fig. 3, the average number of blind attempts according to our schemes in our simulated system is larger than that predicted by (6). This is due to the assumptions that we made along the way to simplify the model. More precisely, the amount of time that a terminal stays in the active state is longer than the average predicted time due to larger packet sizes and the scarcity of radio resources. That is, a terminal despite being in the active state (i.e. awaiting a reception of payload data), may not be scheduled due to the fact that other terminals have higher priorities, but it needs to perform a blind search attempt in this case too. In other words, a terminal performs more blind attempts on the average than that predicted by (6). However, the difference is not large 


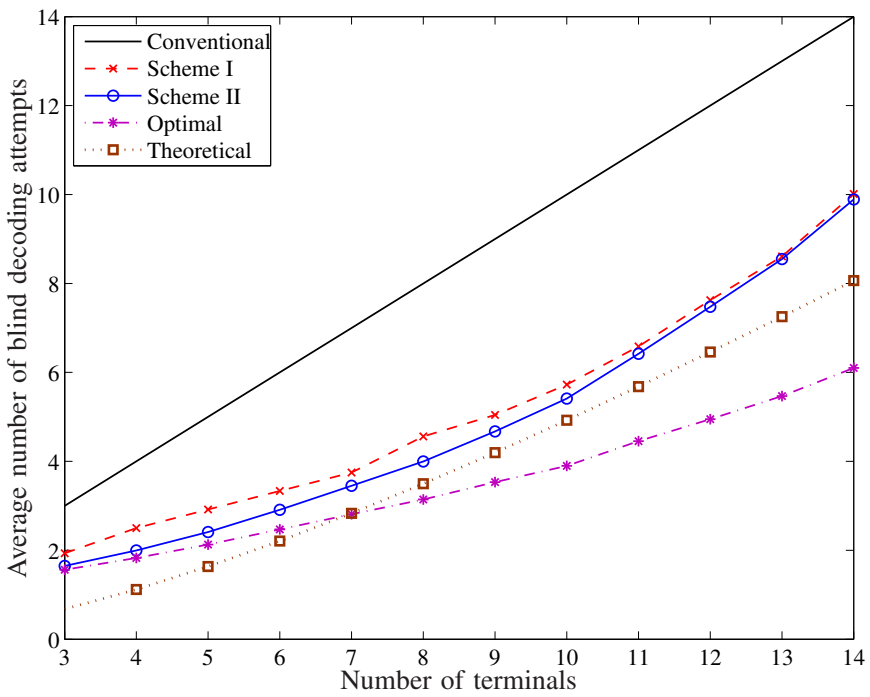

Fig. 3. Average number of blind attempts according to different schemes.

compared to the conventional scheme. The above discussion also explains why the predicted savings is better than the empirical optimum number of blind attempts obtained from system simulations, for small values of $N_{u}$. Also, as we can see, the second scheme is slightly better than the first scheme in terms of average number of blind decoding attempts. This is also predictable since the incoming traffic has a burst behavior.

\section{CONCLUSION}

In this paper, we introduced an idea for reducing the number of blind attempts performed by terminals in order to find their control information. In order for our scheme to work, a "please-decode-blindly" message needs to be broadcast at the beginning of each subframe to instruct a subset of the terminals to perform the blind search. While it is desirable that this subset contains only the scheduled terminals, we showed that this is not possible in practice. The main difficulties are that (i) the set of the scheduled terminals is not fixed, and (ii) the grouping should be done in such a way that no additional signaling is required. Thus, we need to find appropriate grouping strategies, based on the factors such as the available scheduling resources, the traffic behavior of the system, and the number of users. We proposed two schemes to implement our idea and we found the savings of our proposed schemes for a system with a simple model. The numerical results also supported our founding.

The extension of our work should include the study of other grouping strategies. As another extension of our work, one may consider the scheduling problem that achieves a certain quality of service (QoS) while at the same time also takes the number of blind attempts into account. That is, we are interested in distributing the radio resources among the terminals such that the number of blind search attempts that is needed by the terminals is minimized while at the same time we maintain a certain QoS level.

\section{REFERENCES}

[1] E. Dahlman, S. Parkvall and J. Sköld, 4G LTE/LTE-Advanced for Mobile Broadband, 1st edition Academic Press, 2011.

[2] J. Gross, H. F. Geerdes, H. Karl and A. Wolisz, "Performance analysis of dynamic OFDMA systems with inband signaling," IEEE J. Select. Areas Commun., vol. 24, pp. 427-436, Mar. 2006.

[3] E. G. Larsson, "Optimal OFDMA downlink scheduling under a control signaling cost constraint", IEEE Trans. Commun., vol. 58, pp. 2776-2781, Sep. 2010.

[4] R. Moosavi, J. Eriksson, E. G. Larsson, N. Wiberg, P. Frenger and F. Gunnarsson, "Comparison of strategies for signaling of scheduling assignments in wireless OFDMA," IEEE Trans. Veh. Technol., vol. 59, pp. 4527-4542, Nov. 2010 .

[5] D. P. Malladi, J. Montojo and S. Sarkar, Methods and systems for PDCCH blind decoding in mobile communications, United States Patent Application Publication 2009/0168922, Jul. 2, 2009.

[6] A. Reial, L. Andersson, HS-PDSCH blind decoding, United States Patent Application Publication 2009/003301, Jan. 1, 2009.

[7] R. Moosavi and E. G. Larsson, "A fast scheme for blind identification of channel codes," in Proc. of IEEE Global Telecommunications Conference (GLOBECOM), Dec. 2011.

[8] J. F. Cheng, System and method for removing PDCCH detection errors in a telecommunications network, United States Patent Application Publication 2010/0050059, Feb. 25, 2010

[9] J. Eriksson, R. Moosavi and E. G. Larsson, "Complexity reduction of blind decoding schemes using CRC splitting,", in Proc. of IEEE Global Telecommunications Conference (GLOBECOM), Dec. 2012.

[10] X. Qiu and K. Chawla, "On the performance of adaptive modulation in cellular systems," IEEE Trans. Commun., vol. 47, pp. 884-895, Jun. 1999.

[11] 3GPP2 TSG-C WG 3, "cdma2000 Evaluation Methodology V6," Dec. 2006.

[12] C. E. Huang and C. Leung, "QoS-aware bit scheduling in multiuser OFDM systems," in Proc. of IEEE Wireless Communications and Networking Conference (WCNC), May 2011. 